\title{
Epidemiological Investigation and Analysis of Anthrax Epidemic in Jingxi City Guangxi from 2000 to 2020
}

\author{
Wang Fuchun \\ Jingxi City Center for Disease Control and Prevention, Jingxi, China \\ Email address: \\ jxcdc2376@163.com \\ To cite this article: \\ Wang Fuchun. Epidemiological Investigation and Analysis of Anthrax Epidemic in Jingxi City Guangxi from 2000 to 2020. International \\ Journal of Animal Science and Technology. Vol. 5, No. 1, 2021, pp. 13-18. doi: 10.11648/j.ijast.20210501.13
}

Received: January 27, 2021; Accepted: February 9, 2021; Published: February 23, 2021

\begin{abstract}
Objective Analyze the epidemiological characteristics of anthrax in Jingxi City, Guangxi, and Provide a Scientific basis for formulating anthrax Prevention and control measures in the future. Methods Through the National Disease Surveillance Information Management System, collect anthrax epidemic surveillance data in Jingxi City from 2000 to 2020 , and use descriptive epidemiological methods for statistical analysis. Results A total of 18 anthrax cases were reported in the city from 2000 to 2020, including 1 death, with an average annual incidence rate of 0.19 per 100, 000 and a case fatality rate of 5. 56\%; the onset time was concentrated from April to August, with 17 cases reported; There were case reports in 5 towns including zhong longlin Town, Quyang Town, xinjia Township, kuixu Township and Ludong Town in 19 towns in the City, including 5 cases in longlin Town, Quyang Town and xinjia Township (I case of anthrax death in Longlin Town), the number of cases in kuixu Township and Ludong Township were 2 and 1 respectively. There were 16 males and 2 females. The ratio of male to female was 8: 1 ; 16 cases occurred between the ages of 30 and 69 , accounting for $88.89 \%$. Occupationlly, 17 cases of farmers were the most common, Accounting for $94.44 \%$, The main routes of infection were slaughter, Processing, and stripping. There were 16 cases of infected Persons who were directly contacted by animal husbandry who died of food, Sickness and livestock, accounting for 88. $89 \%$. Conclusion The anthrax epidemic in Jingxi City is mainly sporadic, and the population is mainly young and middle-aged and farmers. In the future, it is necessary to strengthen the surveillance of anthrax epidemic, and immunize high-risk Populations in key areas and susceptible animals such as horses, cattle, pigs and livestock. Effectively Prevent and control the occurrence of anthrax.
\end{abstract}

Keywords: Anthrax, Epidemiological Investigation, Analysis

\section{Introduction}

Anthrax is a zoonotic natural infectious disease caused by Bcillus anthracis, with endemic characteristics [1]. Antrax has been one of the most important acute ifectious diseases that seriously endanger the health of humans and animals in Jingxi City, Guangxi for many years. Since the1980s and 1990s, Jingxi City has been a high-incidence area of anthrax in Baise City, Guangxi. The incidence showed a downward trend affer 2000 , but it is still one of the counties with a high incidence of anthrax, In order to understand the characteristics of anthrax fiuidity in Jingxi City, and to provide a reference for formulating anthrax prevention and control measures, the analysis of the anthrax epidemic monitoring data in Jingxi City from 2000 to 2020 is as follows.

\subsection{Background}

Guangxi Jingxi City is located on the Sino-Vietnamese border with a border line of $152.5 \mathrm{~km}$ in the South and Vietnam. It is a subtropical area with a warm and humid climate. The average altitude is above $750 \mathrm{~m}$ and the annual average temperature is $19.1^{\circ} \mathrm{C}$, It has 8 townships and 11 towns with a total population of 670,000 . There are 11 ethnic groups including Zhuang, Han, Miao and Yao, among which the minority Zhuang population accounts for $99.40 \%$, The border ports with Vietnam are Longbang Port (national first-class port) and Yuexu Port (national second-class port). Longlin Street, Longlin Town, is a rural market for animal husbandry trade such as horses and cattle, and Dajia street, Xinjia Township, is a rural market for horse meat and other animal husbandry meat sales and trade markets, horses, cattle and other animal 
husbandry or horse meat and other animal husbandry meat markets. The source of this category is Jingxi City and its surrounging counties. The movement of people and Livestock trade are frequent. The livestock farming is dominated by free-range horses, cattle, sheep and captive pigs in rural areas. Since 2000, large-scale livestock breeding households have gradually increased. Most rural residents go out to work to increase their income, their economic and cultural living standards are low, their awareness of hygiene and disease prevention is weak, they still have bad habits of slaughtering, processing, selling, and eating dead animals, animal husbandry and veterinary hygiene and epidemic prevention are weak, population and susceptible animals are prevented vaccination is not implemented.

\subsection{Literature Review}

Anthrax is a natural foci infectious disease caused by Bacillus anthracis, which is common to humans, livestock and wild animals. Humans are infected by contact with diseased livestock and their products or eating meat from diseased livestock, skin necrosis, ulcers. Scorching Extensive edema and toxemia of the scab and surrounding tissues are the main symptoms. If they are not treated promptly and effectively, the mortality rate is high. Anthrax is an ancient disease. It is recorded in the"Huang Di Nei Jing"in china. In 1849, the famous German Scholar first discovered Bacillus anthracis, and then anthrax has spread or broke out many times around the world. Anthrax is distributed all over the world, especially in Pastoral areas such as South America, Asia, and Africa. It is endemic as a natural foci, once a country or region is contaminated by Bacillus anthracis, it is extremely difficult to eliminate. In the contaminated area, Animals will continue to be infected, threatening humans. In recent years, as the processing of animal skins in various countries in the world is concentrated in cities and towns, anthrax has also broken out in cities, becoming one of the important occupational diseases. According to statistics, there are currently 20,000 to 100,000 people infected with anthrax every year in the world. The anthrax epidemic in China was severe before 2000. After 2000 , the incidence of anthrax showed a downward trend. The incidence fluctuates between 400 and 1,000 people nationwide. At present, the areas with high incidence of anthrax are mainly concentrated in the northwest and southwest regions, Guizhou, xinjiang, Sichuan, Yunnan. The incidence in Guangxi, Qinghai, Xizang, Gansu, Inner Mongolia and other Provinces (regions) accounted for more than $90 \%$ of the total incidence in the country. Since Guangxi included anthrax in the epidemic report in 1956, anthrax has occurred every year, ranking thrid and fourth in the country, Since 2000, the anthrax epidemic has been mainly sporadic and the number of cases has decreased significantly.

\section{Materials and Methods}

\subsection{Data Sources}

Through the "National Disease Surveillance Information
Reporting Management System", and "Public Health Hmergency Reporting Management Information System", the monitoring data of anthrax epidemics in Jingxi City from 2000 to 2020 were collected. The $2000-2020$ Population data comes from Jingxi Municipal Statistics Bureau.

\subsection{Case Diagnosis}

According to the "Anthrax Diagnostic Criteria" of the Ministry of Health, case diagnosis and epidemiological investigation, monitoring and treatment are carried out [2].

\subsection{Methods}

The descriptive epidemiological method was used to collate, count and analyze the anthrax epidemic data in Jingxi City from 2000 to 2020 .

\section{Results}

\subsection{Epidemic Situation}

A total of 18 anthrax cases were reported in Jingxi City from 2000 to 2020 , With an average annual incidence rate of 0.13 Per $100,000,1$ death, and a case fatality rate of $5.56 \%$. In 21 years, only 2004 (7 cases) and 2007 (3 cases), 2008 (4 cases), 2012 (1 cases), 2016 ( 3 cases, including 1 death), there were case reports, and the annual incidence rates were $1.19 / 100,000,0.49 / 100,000$, and $0.64 / 100,000,0.15 / 100,000$, $0.45 / 100,000$, see Table 1 .

\subsection{Time Distrbution}

The 18 anthrax cases were mainly distributed from April to August, including 3 cases in April, 5 cases in May (1 death), 2 cases in July and August, and 1 cases was reported in January. See Table 2.

\subsection{Regional Distribution}

The number of reported cases in Zhonglonglin Town, Quyang Town, and xinjia Township in the 19 towns under the jurisdiction of Jingxi City is 5, the number of reported cases in kuixu Township is 2, and the number of reported cases in Ludong Town is 1 cases. See Table 3.

\subsection{Population Distribution}

The youngest age of onset is 9 years old and the oldest is 74 years old, mainly in the $30-69$ years old (16 cases). There are 16 males and 2 females. The male to female ratio is $8: 1$. The occupation distribution includes 17 farmers and 1 primary school student. See Table 4.

\subsection{Route of Infection}

In the 18 cases of anthrax by the route of infection, 17 cases were clinically diagnosed as skin anthrax, accounting for $94.44 \%$, and 1 suspected case of anthrax died, accounting for $5.56 \%$; the route of infection was direct contact (slaughter, processing, stripping), 16 dead livestock, Accounted for 
88.89\%. 2 cases had indirect contact with sick and dead livestock, accounting for $11.11 \%$, See Table 5 .

Table 1. The report of anthrax cases from 2000 to 2020 in Jingxi City, Guangxi.

\begin{tabular}{llllll}
\hline Years & Total Population & Number of cases & Incidence rate (1/100,000) & death Toll & Case fatality rate (\%) \\
\hline 2000 & 385968 & 0 & 0.00 & 0 & 0.00 \\
2001 & 391225 & 0 & 0.00 & 0 & 0.00 \\
2002 & 392267 & 0 & 0.00 & 0 & 0.00 \\
2003 & 394914 & 0 & 0.00 & 0 & 0.00 \\
2004 & 399199 & 7 & 1.75 & 0 & 0.00 \\
2005 & 401602 & 0 & 0.00 & 0 & 0.00 \\
2006 & 408456 & 0 & 0.00 & 0 & 0.00 \\
2007 & 416967 & 3 & 0.71 & 0 & 0.00 \\
2008 & 429378 & 4 & 0.93 & 0 & 0.00 \\
2009 & 443607 & 0 & 0.00 & 0 & 0.00 \\
2010 & 458504 & 0 & 0.00 & 0 & 0.00 \\
2011 & 464503 & 0 & 0.00 & 0 & 0.00 \\
2012 & 465854 & 1 & 0.21 & 0 & 0.00 \\
2013 & 472165 & 0 & 0.00 & 0 & 0.00 \\
2014 & 472226 & 0 & 0.00 & 1 & 0.00 \\
2015 & 472490 & 0 & 0.00 & 0 & 33.33 \\
2016 & 472797 & 3 & 0.63 & 0 & 0.00 \\
2017 & 472917 & 0 & 0.00 & 0 & 0.00 \\
2018 & 476691 & 0 & 0.00 & 0 & 0.00 \\
2019 & 478072 & 0 & 0.00 & 1 & 0.00 \\
2020 & 486079 & 0 & 0.00 & 0.19 & 5.56 \\
Total & 9255881 & 18 & & 0 &
\end{tabular}

Table 2. The time distribution of anthrax cases from 2000 to 2020 in Jingxi City, Cruangxi.

\begin{tabular}{|c|c|c|c|c|c|c|c|c|c|c|c|c|c|}
\hline Month & 1 & 2 & 3 & 4 & 5 & 6 & 7 & 8 & 9 & 10 & 11 & 12 & Total \\
\hline Number of cases & 1 & 0 & 0 & 3 & 5 & 5 & 2 & 2 & 0 & 0 & 0 & 0 & 18 \\
\hline death toll & 0 & 0 & 0 & 0 & 0 & 1 & 0 & 0 & 0 & 0 & 0 & 0 & 1 \\
\hline Composition ratio $(\%)$ & 5.56 & 0.00 & 0.00 & 16.67 & 27.78 & 27.78 & 11.11 & 11.11 & 0.00 & 0.00 & 0.00 & 0.00 & 100.00 \\
\hline
\end{tabular}

Table 3. The regional distribution fo anthrax cases from 2000 to 2020 in Jingxi city, Guangxi.

\begin{tabular}{llllll}
\hline Township & Average annual population & Number of cases & Incidence rate (1/100,000) & Death toll & Case fatalityrate (\%) \\
\hline Xingjing & 1358282 & 0 & 0 & 0 & 0 \\
Huadong & 310381 & 0 & 0 & 0 & 0 \\
Tongde & 426301 & 0 & 0 & 0 & 0 \\
Hurun & 355951 & 0 & 0 & 0 & 0 \\
Yuexu & 220059 & 0 & 0 & 0 & 0 \\
Renzhuang & 291606 & 0 & 0 & 0 & 0 \\
Dizhou & 358890 & 0 & 0 & 0 & 0 \\
Longbang & 287112 & 0 & 0 & 0 & 0 \\
Anning & 234864 & 0 & 0 & 0 & 0 \\
Ludong & 733929 & 1 & 0.13 & 0 & 0 \\
Tunpan & 291795 & 0 & 0 & 0 & 0 \\
Nanpo & 475272 & 0 & 0 & 0 & 0 \\
Ande & 646653 & 0 & 0 & 0 & 0 \\
Longlin & 589134 & 5 & 0.84 & 1 & 20.00 \\
Guole & 456771 & 0 & 0 & 0 & 0 \\
Xinjia & 608601 & 5 & 0.82 & 0 & 0 \\
Quyang & 604758 & 5 & 0.82 & 0 & 0 \\
Kuixu & 338436 & 2 & 0.59 & 0 & 0 \\
Wuping & 667086 & 0 & 0 & 0 & 0 \\
Total & 9255881 & 18 & 0.19 & 1 & 0 \\
\hline
\end{tabular}


Table 4. The ropulation distribution of anthrax cases from 2000 to 2020 in Jingxi City, Guangxi.

\begin{tabular}{|c|c|c|c|c|c|c|c|}
\hline \multirow{2}{*}{ Age } & \multirow{2}{*}{$\begin{array}{l}\text { Number } \\
\text { of cases }\end{array}$} & \multicolumn{2}{|c|}{ Number of cases } & \multicolumn{2}{|c|}{ Death toll } & \multirow{2}{*}{ Composition ratio (\%) } & \multirow{2}{*}{ Case fatality rate (\%) } \\
\hline & & Male & Female & Male & Female & & \\
\hline 0 & 0 & 0 & 0 & 0 & 0 & 0.00 & 0.00 \\
\hline 5 & 1 & 0 & 1 & 0 & 0 & 5.56 & 0.00 \\
\hline 10 & 0 & 0 & 0 & 0 & 0 & 0.00 & 0.00 \\
\hline 20 & 0 & 0 & 0 & 0 & 0 & 0.00 & 0.00 \\
\hline 30 & 6 & 6 & 0 & 0 & 0 & 33.33 & 0.00 \\
\hline 40 & 6 & 6 & 0 & 1 & 0 & 33.33 & 16.67 \\
\hline 50 & 2 & 2 & 0 & 0 & 0 & 11.11 & 0.00 \\
\hline 60 & 2 & 2 & 0 & 0 & 0 & 11.11 & 0.00 \\
\hline 70 & 1 & 0 & 1 & 0 & 0 & 5.56 & 0.00 \\
\hline$\geq 80$ & 0 & 0 & 0 & 0 & 0 & 0.00 & 0.00 \\
\hline Total & 18 & 16 & 2 & 1 & 0 & 100.00 & 5.56 \\
\hline
\end{tabular}

Table 5. The route of infection of anthrax cases from 2000 to 2020 in Jingxi City, Guangxi.

\begin{tabular}{lllllll}
\hline \multirow{2}{*}{ Years } & \multirow{2}{*}{ Number of cases } & Death toll & Route of infection & & & \\
\cline { 3 - 6 } & & Direct contact & Indirect Contact & Processed fur & Inhalation of Dust, aerosol \\
\hline 2000 & 0 & 0 & 0 & 0 & 0 & 0 \\
2001 & 0 & 0 & 0 & 0 & 0 & 0 \\
2002 & 0 & 0 & 0 & 0 & 0 & 0 \\
2003 & 0 & 0 & 0 & 0 & 0 & 0 \\
2004 & 7 & 0 & 6 & 1 & 0 & 0 \\
2005 & 0 & 0 & 0 & 0 & 0 & 0 \\
2006 & 0 & 0 & 0 & 0 & 0 & 0 \\
2007 & 3 & 0 & 3 & 0 & 0 & 0 \\
2008 & 4 & 0 & 1 & 0 & 0 \\
2009 & 0 & 0 & 0 & 0 & 0 & 0 \\
2010 & 0 & 0 & 0 & 0 & 0 & 0 \\
2011 & 0 & 0 & 0 & 0 & 0 & 0 \\
2012 & 1 & 0 & 1 & 0 & 0 & 0 \\
2013 & 0 & 0 & 0 & 0 & 0 & 0 \\
2014 & 0 & 0 & 0 & 0 & 0 & 0 \\
2015 & 0 & 0 & 0 & 0 & 0 & 0 \\
2016 & 3 & 1 & 3 & 0 & 0 & 0 \\
2017 & 0 & 0 & 0 & 0 & 0 & 0 \\
2018 & 0 & 0 & 0 & 0 & 0 & 0 \\
2019 & 0 & 0 & 0 & 0 & 0 & 0 \\
2020 & 0 & 0 & 0 & 2 & & 0 \\
Total & 18 & 1 & 16 & & 0 & 0 \\
\hline
\end{tabular}

\subsection{Types of Clinical Infections}

The clinical infection type of anthrax patients is mainly skin anthrax (17 cases), accounting for $94.44 \%$, Suspected anthrax case death ( 1 cases), accounting for $5.56 \%$, and the distribution period of incubation period is 1 to 5 days, there are 15 case, accounting for $83.33 \%, 6$ to 8 days 3 cases, accounting for $16.67 \%$.

\section{Discussion}

The analysis of the monitoring data of the anthrax epidemic report in Jingxi City of Guangxi in the 21 st century showed that the City's anthrax epidemics occurred from time to time, mainly sporadic, with occasional outbreaks in local villages [3]. The main source of infection of anthrax in the City is herbivores horses, sheep or livestock Pigs. The high-risk Populations susceptible to anthrax are mainly rural residents who have come into contact with dead animals in natural epidemic areas, especially those involved in the slaughter, Processing, Stripping of dead animals, or those engaged in breeding. The average incidence of anthrax in 21 years was 0.19 per 100,000 . There were no reports of anthrax cases in 2000-2003, 2005-2006, 2009-2011, 2013-2015, 2017, 2020. The rest of the years $(2004,2007$, 2012, 2016).

A total of 18 cases of anthrax have been reported. The population is mainly male young adults and farmers aged 30-69. The onset season is mainly from April to August each year. The affected areas are mainly distributed in the natural foci of the old anthrax epidemic a rea. The main method of transmission is through direct contact with slaughter, processing, and animal husbandry.

There were 3 reported cases of anthrax in 2016, including 1 
suspected anthrax death. The case died at home in a rural area due to not being admitted to the hospital in time. The time from onset to death was 3-4 hours due to lack of hospital clinical diagnosis and laboratory testing. According to the data, after consultation with relevant disease control medical experts from Guangxi province, Baise City, Jingxi City centers for Disease control and prevention and Baise City people's Hospital, it was diagnosed as a suspected case of anthrax. The possibility of death due to cardiogenic shock is high [4]. The clinical infection type of anthrax patients is mainly skin anthrax, and the incubation period of patients is 1 to 5 days. This is basically consistent with previous reports in china [5-8].

Anthrax mainly occurs in animal husbandry. Horses, cattle, sheep, camels and pigs are the most susceptible. Herbivores or livestock are in contact with, eating or drinking forage and water contaminated by Bacillus anthracis, and inhalation contains anthrax spores, Bacillus dust or aerosol can cause anthracnose among livestock [9-11].

Humans are generally susceptible to anthrax, usually due to (slaughter, processing, stripping) contact with diseased livestock and their products or eating meat from diseased livestock [12].

The occurrence of the anthrax epidemic in Jingxi City, Guangxi may be related to the following factors: (1) some towns in the city are natural foci of anthrax. Because of the strong resistance of Bacillus anthracis, it has long survived in the soil, water plants, trees and dead animal bones. Infection after contact with livestock becomes the source of anthrax infection [13-14]. (2) The city is an ethnic minority area and a sino-vietnamese border area. The economy and culture are relatively underdeveloped, the living standards of rural residents are still low, the awareness of health problems is not high, and the awareness of the serious harm of anthrax is insufflcient, and rural residents still have direct contact (slaughter, processing, stripping) The bad habits of sick and dead animal husbandry, lack of effective personal Protection measures, resulting in direct or indirect cross-infection with Bacillus anthracis when exposed to broken skin, wounds or knives during the process of slaughtering, processing, and stripping sick and dead animal husbandry, causing cases of skin anthrax or intestinal anthrax. (3) The joint prevention and control mechanism of agricultural and rural animal husbandry and veterinarians, market supervision, food safety, and sanitation and health primary health institutions and other departments is not complete. In terms of livestock, anthrax vaccination of livestock animals in anthrax epidemic areas has not been fully implemented, and the epidemic report is not timely, strict disinfection and other measures are not implemented, animal husbandry and veterinary inspection and quarantine supervision and disposal are not strict, and the slaughter of sick and dead animals has not been banned and effectively and strictly disinfected, burned, and buried. (4) There are still blank susceptibility risks and weak links in anthrax vaccination for high-risk populations in towns and towns where anthrax occurs.

In order to effectively prevent and control the spread of anthrax, a government-led joint prevention and control mechanism should be established and improved, and communication and coordination between agricultural and rural animal husbandry and veterinary, market supervision and food safety, health and health basic-level health institutions and other departments should be strengthened, and the relevant departments of each department should be actively performed; carry out anthrax epidemic monitoring, especially do a good job in the monitoring of animal husbandry and human anthrax epidemics in anthrax-affected areas, strengthen social publicity and education, raise the people's awareness of disease prevention, and make the people consciously achieve the "three in one persistence", that is, to do the same for anthrax animals No slaughter, no stripping, no processing and sales, adhere to effective and thorough disinfection, incineration, and deep burying of anthrax animal carcasses, and immunize high-risk populations and susceptible animals such as horses, cattle and pigs in epidemic areas to prevent and control anthrax epidemics The occurrence of [15].

\section{Conclusion}

The anthrax epidemic in Jingxi City, Guangxi is mainly sporadic. The infection rate of young adults and farmers in rural areas are high. Human anthrax is mainly infected by contact with livestock anthrax. Epidemic Surveillance should be strengthened, animal husbandry and veterinary inspection and quarantine mechanisms should be established, and all sick and dead animals should be disinfected, burned, and buried deeply; anthrax vaccination should be carried out for high-risk populations in epidemic areas and susceptible animals such as horses, cattle, and pigs to control the source of infection, cut off the transmission route, the fundamental measure to effectively prevent and control the anthrax epidemic.

\section{References}

[1] Li Lanjuan, Ren hong, Infectious Diseases, 8rd ed, Beijing: people's health, 2015, PP. 193-196.

[2] Ministry of Health of the people's Republic of china, ws 283-2008 Diagnostic criteria for anthrax, Beijing: people's health, 2008.

[3] Wang Guoqiang, 60 years of disease prevention and control in china, Beijing: China entrance, 2015, pp. 177-180.

[4] Wang Fuchun (2016), Epidemiological investigation and analysis of an anthrax epidemic in Jingxi City, Gunagxi, Public Health and preventive Medicine, 27 (6): 91-93.

[5] Ren Liyun (2015), Epidemiological investigation of cutaneous anthrax, 6, 28-30.

[6] Meng Linming, Xu Yuanpeng, He Fenyi, et al (2014). Epidemic characteristics of zoonotic diseases and suggestions for pervention and control, Chinese Veterinary Journal, 15 (12): $37-38$ 
[7] Ma Qing, Chen Hong, Liu Ying, et al (2016). Eiological analysis of an outbreak of skin anthrax in Guizhou Province, Heilongjiang Animal Husbandry and veterinary, 41 (2): 94-96.

[8] Zhang wenjing, LiQinghua, Wang Yong, et al (2013). epidemiological andlysis of skin anthrax in china from 2007 to 2011, Military medicine, 37 (12): 892-894.

[9] Han guohua, Nan Jinyu, Liu Zhanqi, et al (2015). Diagnosis, investigation and eradication of a case of swine anthracnose, Journal of Animal Husbandry and veterinary Medicine, 34 (5): 126-127.

[10] Li Xiuying, Wang Yunping, Wang Shengxiang (2014). Epidemiological Survey of anthracnose among Livestock in Qinghai, chinese veterinary Journal, 50 (6): 74-75.

[11] Lu Chunli, Qin Yong, Meng Zhemu (2015). Epidemiological investigation and analysis of anthrax epidemic situation in Baise, Guangxi and its control strategies, chinese veterinary Journal, 51 (6): 88-90.

[12] Shi Dengquan (2014). Investigation of an anthrax epidemic in Rongjiang county, Guizhou province, Modern preventive medicine, 41 (2): 351-352.

[13] Wang Fuchun, Huang Wenxian, Wei Bin (2017). Analysis on the characteristics of anthrax fluidity in Jingxi City, Guangxi from 2004 t0 2015, south china preventive Medicine, 43 (2): 148-149.

[14] Wu Jina (2014). Investigation report of an outbreak of anthacnose, contemporary Medical Essays, 12 (5): 286-287.

[15] Xie Gang, Zhang Guoming, Wen Nianping, et al (2014). Epidemiological characteristics of anthrax in Hong yuan county from 2005 to 2011, Journal of preventive Medicine Information, 30 (2): 114-116. 